\title{
Manifestaciones clínicas y complicaciones de la Enfermedad por Coronavirus 2019 (COVID-19): revisión de la literatura
}

\author{
Clinical manifestations and complications of Coronavirus Disease 2019 (COVID-19) \\ Herberth Maldonado Briones ${ }^{1,2,3}$, Mario Melgar Toledo ${ }^{3,4,5}$, \\ Nancy Sandoval Paiz ${ }^{5,6}$, Hugo Pezzarossi Zelaya ${ }^{7}$ \\ ${ }^{1}$ Centro de Estudios en Salud, Universidad del Valle de Guatemala, ${ }^{2}$ Unidad de Cirugía Cardiovascular de Guatemala, \\ ${ }^{3}$ Facultad de Ciencias Médicas, Universidad San Carlos de Guatemala, ${ }^{4}$ Unidad de Oncología Pediátrica, Guatemala, \\ ${ }^{5}$ Hospital Roosevelt, Guatemala, ${ }^{6}$ Facultad de Medicina, Universidad Rafael Landívar, Guatemala, \\ ${ }^{7}$ Medicina Interna y Enfermedades Infecciosas, Hospital El Pilar, Guatemala \\ *Autor al que se dirige la correspondencia: hmaldonado@ces.uvg.edu.gt
}

\section{Resumen}

Recibido: 06 de septiembre 2020 / Revisión: 20 de septiembre 2020 / Aceptado: 12 de octubre 2020

\begin{abstract}
T a enfermedad por coronavirus 2019 (COVID-19) supone un reto sin precedentes para la salud pública. Los médicos deben fortalecer sus habilidades clínicas para combatir esta nueva enfermedad. El objetivo de esta revisión fue describir los signos, síntomas y complicaciones de pacientes con COVID-19. Se realizó una búsqueda en la literatura en la base de datos PubMed incluyendo publicaciones entre 1 de diciembre 2019 y 15 de agosto 2020, sin restricciones de lenguaje, utilizando las palabras clave 'COVID-19' cruzada con 'manifestaciones clínicas', 'signos y síntomas' y 'complicaciones'. Se incluyeron revisiones sistemáticas y meta-análisis, estudios epidemiológicos y series de casos. Se identificaron 1,066 artículos, de los cuales se seleccionaron 71 de acuerdo a los contenidos previamente definidos por los autores. La COVID-19 presenta un curso de leve a moderado y severo-crítico, específicamente en mayores de 65 años con o sin comorbilidades. Los signos y síntomas no son específicos y usualmente se superponen de acuerdo a la edad y a la fase de la enfermedad. Los síntomas más comunes son fiebre, tos y fatiga. Síntomas menos comunes incluyen escalofríos, dolor de garganta, cefalea, mialgias/artralgias, pérdida del gusto y del olfato, diarrea, nausea, vómitos, congestión nasal, palpitaciones, opresión precordial y dolor pleurítico. La sintomatología en niños difiere de la de los adultos y el curso es usualmente benigno y de baja mortalidad. La evaluación clínica de COVID-19 supone un reto hoy en día. Sin embargo, el conocimiento de la historia natural de la enfermedad permite situar los síntomas comunes e inespecíficos en el contexto clínico correcto.
\end{abstract}

Palabras claves: COVID-19, SARS-CoV-2, signos, síntomas, complicaciones

Abstract

$\mathrm{C}$ oronavirus disease 2019 (COVID-19) presents an unprecedented challenge to public health. Physicians must strengthen clinical skills for this new disease. This review aimed to describe the signs, symptoms and complications of patients with COVID-19. A literature search was conducted in the PubMed database, including publications between December 1, 2019, to August 15, 2020, without language restrictions, using the keywords 'COVID-19' crossed with 'clinical manifestations', 'signs and symptoms', and 'complications'. Systematic reviews, meta-analysis, epidemiological studies, and case report series were included. A total of 1,066 articles were identified, among 71 were selected according to the contents previously defined by the authors. COVID-19 has a mild to moderate or severe-critical course, specifically in over 65 years of age, with or without comorbidities. Signs and symptoms are not specific and usually overlap according to the age and stage of the disease. The most common symptoms are fever, cough, and fatigue. Less common symptoms include chills, sore throat, headache, myalgia/ arthralgia, loss of taste and smell, diarrhea, nausea, vomiting, nasal congestion, palpitations, chest tightness, and pleuritic pain. The symptomatology in children differs from that of adults, and the course is usually benign and low mortality. Clinical evaluation of COVID-19 is challenging today. However, knowledge of the disease's natural history allows placing common and nonspecific symptoms in the correct clinical context.

Keywords: COVID-19, SARS-CoV-2, signs, symptoms, complications 


\section{Introducción}

En diciembre 2019 se reportaron en Wuhan, China los primeros casos del coronavirus de tipo 2 causante del síndrome respiratorio agudo severo (SARS-CoV-2), causante de la enfermedad por coronavirus 2019 (COVID-19), que se ha propagado a otros países y continentes, siendo declarada ta pandemia el 11 de marzo de 2020 por la Organización Mundial de la Salud (Cucinotta \& Vanelli, 2020). El primer caso se registró en Guatemala el 13 de marzo de 2020 y al 30 de septiembre se reportan 92,408 casos y 3,453 decesos, con una incidencia acumulada de 548 casos por 100,000 habitantes (Ministerio de Salud Pública y Asistencia Social [MSPAS], 2020a).

Dado que la COVID-19 se considera endémica hasta la aparición de una vacuna efectiva, los médicos deben fortalecer el conocimiento para esta nueva enfermedad. Los signos y síntomas de COVID-19 son inespecíficos, sin embargo los médicos afinarán su capacidad de diagnóstico conforme la creciente experiencia en la clínica.

El abordaje de COVID-19 supone un reto importante para los médicos y proveedores de salud en nuestro país, pues existe escaso recurso humano y el acceso es limitado a pruebas diagnósticas confiables que detecten ácidos nucleicos como la reacción en cadena de polimerasa de transcriptasa reversa (RT-PCR). En relación al recurso humano, Guatemala reporta apenas 2.8 médicos por cada 10,000 habitantes, el segundo más bajo de las Américas, encontrándose cuatro y ocho veces por debajo del promedio del istmo centroamericano y latinoamericano respectivamente (Organización Panamericana de la Salud [OPS], 2019).

Luego de más de ocho meses de creciente conocimiento sobre esta nueva enfermedad, presentamos esta revisión de literatura con el propósito de proporcionar información clínica relevante y sintetizada, que pueda ser utilizada por el clínico para su apoyo durante el abordaje diario de pacientes con sospecha de COVID-19. La revisión fue realizada en PubMed entre 1 de diciembre de 2019 y 15 de agosto de 2020 cruzada con 'manifestaciones clínicas', 'signos y síntomas' y 'complicaciones'. Se incluyeron revisiones sistemáticas y meta-análisis, estudios epidemiológicos y series de casos. Se identificaron 1,066 artículos, de los cuales se seleccionaron 71 de acuerdo a los contenidos previamente definidos por los autores.

\section{Contenido}

\section{Ruta de transmisión}

El virus SARS-CoV-2 se transmite de persona a persona, la principal ruta de transmisión es por vía aérea luego de la inhalación de partículas virales presentes en núcleos de gotitas o aerosoles. Las gotitas se generan cuando personas enfermas tosen o estornudan, alcanzando una distancia de hasta 2 por efecto de la gravedad, mientras que los aerosoles $(<5$ micras) permanecen en el ambiente por algunas horas (Klompas et al., 2020). Otra importante ruta de transmisión es a través del contacto directo con la mucosa ocular, oral y nasal, generalmente a través de la autoinoculación de las manos contaminadas que han tocado superficies contaminadas. La vía fecal-oral y la vía vertical madre-hijo también han sido descritas aunque su papel es menor en la diseminación de la enfermedad en seres humanos (Yang et al., 2020).

\section{Sitio de entrada y el receptor ACE2}

La entrada de los coronavirus se produce cuando su proteína de espiga (S) se une a un receptor celular específico llamado enzima convertidora de angiotensina 2 (ACE2), tras lo cual una proteasa 2 de la serina de la transmembrana (TMPRSS2) facilita su ingreso a la célula alcanzada. La mayor co-expresión de ACE2 y TMPRSS2 se encuentra en las células caliciformes o de goblet nasales y las células ciliares del aparato respiratorio. También se expresan en la córnea, esófago, íleo, colon, hígado, vesícula biliar, corazón, riñón y testículo. No se encuentra presencia de ACE2 y TMPRSS2 en el bazo, músculo esquelético, retina, cerebro ni en la piel (Sungnak et al., 2020).

En pacientes infectados con y sin síntomas, los hisopados nasales muestran mayor carga viral que los orofaríngeos, implicando que el epitelio nasal es el principal portal inicial de la infección y transmisión (Zou et al., 2020).

\section{Período de incubación, período de latencia, shedding viral y período de infecciosidad}

El período de incubación (tiempo entre la exposición y la aparición de síntomas), es de 2 a 5 días luego de la exposición, con un rango de 2 a 14 días (Lauer 
et al., 2020). El período de latencia (tiempo entre la infección hasta la capacidad de transmitir) es de 3.69 días (Q. Li et al., 2020 et al., 2020). El shedding es un término acuñado para describir la liberación al medio ambiente de partículas virales desde células infectadas, ya sea a través de secreciones o de líquidos corporales, siendo la duración media de 20 días (RIC 17-24) y la mayor duración hasta 37 días (Zhou et al., 2020). Sin embargo, el período de infecciosidad, durante el cual el individuo permanece infeccioso se ha establecido desde 2 días antes del inicio de síntomas hasta 10 días después, con el pico en tres días y medio (He et al., 2020b).

\section{Factores de riesgo asociados a severidad}

Las condiciones y comorbilidades asociados a severidad y mortalidad son edad avanzada, obesidad, diabetes e hipertensión. La enfermedad severa y crítica está asociada con daño de órganos afectando principalmente el corazón, hígado y riñones. La inflamación y la disfunción de la coagulación también son factores de mal pronóstico (Wolff et al., 2020).

\section{Presentación de la enfermedad}

La infección por SARS-CoV-2 se presenta como una infección asintomática o bien como una enfermedad, -la COVID-19- con un curso leve, moderado, severo y crítico.

Las infecciones asintomáticas representan del 30 al 50\% de los casos, siendo diagnosticados por la detección de ácidos nucleicos virales durante investigaciones de contactos. Aunque no presentan signos ni síntomas, ni anomalías radiológicas aparentes, tienen el potencial de transmisión igual al de aquellos con síntomas. La incidencia de infecciones asintomáticas en niños son menores, posiblemente debido a niveles altos de receptores ACE2 (Gao et al., 2020).

El tiempo de duración de la enfermedad es de dos semanas desde el inicio de síntomas hasta la recuperación para los casos leves y de tres a seis semanas para los casos severos. Las manifestaciones más comunes de COVID-19 son similares al resfriado común, como fiebre, tos seca, disnea y fatiga. Síntomas menos comunes incluyen escalofríos, dolor de garganta, cefalea, mialgia/artralgia, pérdida del gusto y del olfato, diarrea, nausea, vómitos, congestión nasal, palpitaciones, opresión precordial y dolor pleurítico (Goyal et al., 2020; Guan et al., 2020).
En Guatemala, el Departamento de Epidemiología del Ministerio de Salud Pública y Asistencia Social ha informado que en 64,870 casos confirmados de todas las edades los síntomas reportados fueron fiebre $(84 \%)$, tos $(59 \%)$, malestar general $(57 \%)$, cefalea $(53 \%)$, mialgias $(44 \%)$, faringodinia $(32 \%)$, anosmia o disgeusia (30\%), disnea (22\%), rinorrea (21\%) y vómitos (17\%) (MSPAS, 2020).

Se ha propuesto la clasificación de la infección en tres fases que pueden ser progresivas: temprana (I), fase pulmonar con y sin hipoxia (IIa/IIb) y fase de hiperinflamación (III). La fase I corresponde a una replicación viral activa y la presencia de síntomas como fiebre de corta duración, tos y constitucionales leves, linfopenia y usualmente ningún hallazgo radiológico. Luego de cinco a siete días algunos pacientes progresan al estadio II, que corresponde a una enfermedad moderada con neumonía radiológicamente comprobada, disnea y fatiga, acompañada de desaturación $\left(\mathrm{SatO}_{2}<\right.$ $90 \%$ ) e hipoxemia $\left(\mathrm{PaO} 2 / \mathrm{FiO}_{2}<300 \mathrm{mmHg}\right)$. La fase III representa la progresión a un estado hiperinflamatorio que será descrito más adelante, con síndrome de dificultad respiratoria aguda (SDRA), choque cardiogénico, con una elevación importante de biomarcadores inflamatorios, alteraciones de la coagulación (Siddiqi \& Mehra, 2020) como se muestra en la Figura 1.

\section{Evaluación clínica: anamnesis y exploración física}

La anamnesis y el examen físico minuciosos forman la columna vertebral del razonamiento clínico y elaboración del diagnóstico diferencial. Durante la pandemia, los tiempos de evaluación clínica son reducidos o inexistentes (van Dam et al., 2020). La sobrecarga laboral, el distanciamiento físico, así como el uso continuo y a veces excesivo de equipo de protección personal, incluyendo mascarillas de alta eficiencia, protectores faciales, gafas, batas y guantes producen un distanciamiento entre el médico y el paciente (Chow, 2004). El motivo de consulta es la motivación más importante para buscar atención, sin embargo, los pacientes pueden modificar la motivación a consultar por temor a los prejuicios y a la discriminación de estar enfermos de COVID-19. El médico debe ser intuitivo y realizar preguntas primero abiertas y luego cerradas para obtener información relevante sobre los principales síntomas y su aparición. La anamnesis debe incluir los antecedentes médicos, historial de viajes y exposiciones, ocupación laboral, composición del 


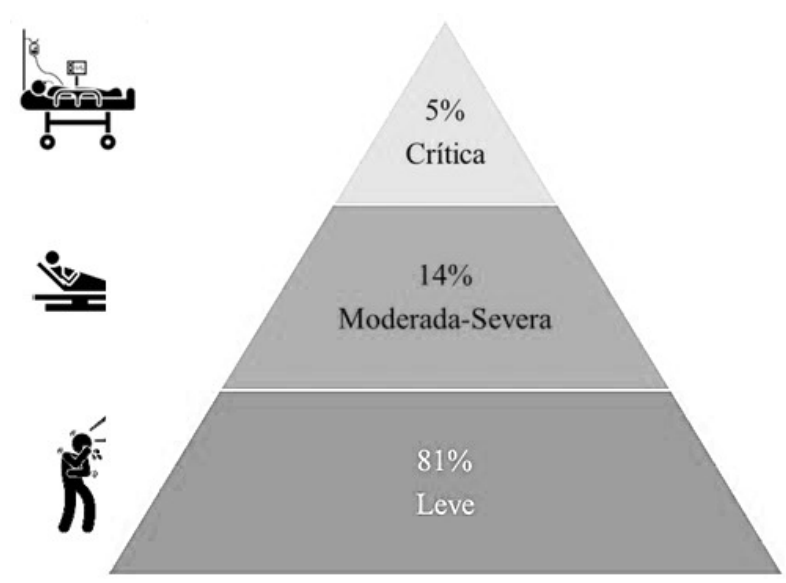

- Insuficiencia respiratoria, $\left(\mathrm{SpO}_{2}\right) \leq 90 \%$

- Choque séptico y/o disfunción orgánica múltiple

- Complicaciones tromboembólicas

- $2.3 \%$ de mortalidad.

- Neumonía y progresión a SDRA: frecuencia respiratoria $>30 / \mathrm{min},\left(\mathrm{SpO}_{2}\right) \leq 93 \%, \mathrm{PaO}_{2} / \mathrm{FiO}_{2}<300 \mathrm{y} / \mathrm{o}$ infiltrados pulmonares $>50 \%$ en 24 a 48 horas.

Síntomas similares a un cuadro gripal

No neumonía o neumonia leve

Recuperación en 2 semanas

Figura 1. Presentación clínica y complicaciones de la COVID-19

núcleo familiar, frecuencia de interacción e historial de signos y síntomas en las últimas dos semanas. El interrogatorio por sistemas debe realizarse de forma cefalocaudal, realizando preguntas directas estructuradas, evitando inducir una respuesta (Cruz et al., 2012). El examen físico debe incluir la toma de signos vitales, incluyendo la oximetría de pulso digital. La exploración física debe ser orientada a la búsqueda de hallazgos relevantes, utilizando guantes y bata impermeable en la exploración de las mucosas y posible exposición a fluidos corporales.

Las prácticas de control y prevención de SARSCoV-2 deben ser implementadas en la clínica e incluyen la higiene de manos en los cinco momentos de atención, el adecuado uso del equipo de protección personal, la limpieza y desinfección frecuente del entorno de atención. Las guías técnicas para su implementación se encuentran disponibles en línea en los sitios de la Organización Mundial de la Salud (WHO, 2020) y de los Centros para el Control de Enfermedades de los Estados Unidos (CDC, 2020).

\section{Manifestaciones oculares}

Se ha reportado la presencia de SARS-CoV-2 por RT-PCR en tejidos y secreciones oculares de enfermos de COVID-19, vía inoculación directa o bien por migración desde la nasofaringe o el tracto lagrimal, pudiendo afectar la conjuntiva, el segmento anterior, retina e incluso el nervio óptico (Seah \& Agrawal, 2020). Entre los síntomas predominan conjuntivitis como ojo rojo, irritación ocular, sensación de cuerpo extraño, lagrimeo y quemosis. A la exploración se encuentran hallazgos de conjuntivitis folicular, inyección conjun- tival unilateral o bilateral y edema leve del párpado. Usualmente los síntomas oculares están asociados a tos y fiebre, con recuperación espontánea (Ma et al., 2020; Wu et al., 2020).

\section{Manifestaciones en aparato respiratorio}

El portal de entrada para la infección y mayor carga viral ocurre en el aparato respiratorio, sin embargo, la presentación clínica de síntomas es variable de acuerdo a la severidad de la enfermedad.

En la vía aérea superior los síntomas más frecuentemente reportados son faringodinia (12.4\%), congestión nasal (3.7\%) y rinorrea (4\%) (Lovato \& de Filippis, 2020). De forma similar a otras neumonías, los síntomas encontrados en pacientes COVID-19 son la fiebre, fatiga y tos seca o productiva, disnea y dolor torácico (D. Zhao et al., 2020). A medida que avanza la enfermedad, se pueden auscultar crepitantes finos, como en la neumonía temprana. Una vez que un paciente ha desarrollado síndrome de dificultad respiratoria aguda (SDRA), se auscultan estertores y roncus difusos (Wang et al., 2020).

Las manifestaciones radiológicas de neumonía viral por COVID-19 más frecuentes son opacidades en vidrio despulido con o sin consolidaciones difusas, de predominio bilateral en las bases y periferia, presentes en hasta la mitad de los pacientes. En menor frecuencia se encuentran broncogramas aéreos $(20 \%)$, patrones reticulares $(14 \%)$, engrosamiento bronquial y pleural (10\%) (Gravell et al., 2020; Z. Ye et al., 2020).

La tomografía computada (TC) tiene una sensibilidad del 93\% para el diagnóstico de neumonía y enfermedad pulmonar, mientras que para la radiografía 
de tórax y el ultrasonido es del $82 \%$ y del $78 \%$ respectivamente. La especificidad de la TC es apenas del 18\%, mientras que no ha sido establecida para la radiografía de tórax y ultrasonido (Lu et al., 2020; Salameh et al., 2020; Wong et al., 2020).

La realización de estudios radiológicos de rutina no está justificada. Para los casos moderados a severos la radiografía de tórax es la modalidad de preferencia dada su mayor disponibilidad y menor costo, siendo posible realizarla con equipos portátiles, debiendo apoyarse en la lectura e interpretación por especialistas en radiología (Jacobi et al., 2020). La TC juega un papel importante en la detección temprana y monitoreo de la evolución de la enfermedad pulmonar, sin embargo presenta un mayor costo y exposición a radiación al paciente, por lo que debe tenerse precauciones en niños y mujeres embarazadas. El ultrasonido pulmonar es una alternativa, sin embargo requiere personal entrenado y mayor tiempo de exposición del operador $(\mathrm{Hu}$ \& Wang, 2020).

\section{Manifestaciones cardíacas y vasculares}

Los pacientes con COVID-19 pueden manifestar signos de enfermedad cardíaca, presentando un síndrome coronario agudo o falla cardíaca congestiva por disfunción ventricular. Los síntomas de un síndrome coronario agudo incluyen dolor precordial, disnea y disfunción del seno que se traslapa con los síntomas de COVID-19. El diagnóstico requiere la elevación de troponina cardíaca y/o anomalías ecocardiográficas y electrocardiográficas. La disfunción ventricular en COVID-19 se presenta con características de falla cardíaca, choque cardiogénico y miocarditis. El diagnóstico se establece al evidenciar la elevación de biomarcadores cardíacos y por hallazgos de carditis en la resonancia magnética cardíaca y biopsia (Sattar et al., 2020). La disfunción eléctrica se manifiesta con arritmias, choque cardiogénico y muerte súbita, teniendo mayor riesgo aquellos pacientes críticamente enfermos con alteraciones metabólicas y múltiples interacciones farmacológicas proarritímicas (Dhakal et al., 2020).

La hipertensión es frecuentemente documentada en pacientes con COVID-19, que se considera producto de una vasoconstricción favorecida por una desregulación del control de angiotensina II, empeorado por una hipertensión subyacente por disfunción endotelial y el estrés oxidativo, que limita la disponibilidad de óxido nítrico y de la inhibición de la actividad enzimática de la óxido nítrico sintetasa (Schiffrin et al., 2020).
La hipercoagulabilidad y enfermedad tromboembólica es otra manifestación vascular multifactorial desencadenada por la tormenta de citocinas, la elevación de angiotensina II, endotelina-1, inhibidor del activador de plasminógeno 1 (PAI-1), factor tisular, especies reactivas de oxígeno y la cascada extrínseca de la coagulación (Connors \& Levy, 2020). El perfil de coagulación de pacientes con COVID-19 muestra trombocitopenia, elevación del dímero-D, tiempo de protrombina prolongado y del INR, así como un tiempo de tromboplastina parcial activada corto (aPTT), predisponiendo al paciente a complicaciones trombóticas (Bikdeli et al., 2020).

\section{Manifestaciones gastrointestinales}

El SARS-CoV-2 puede infectar células del epitelio intestinal vía el receptor ACE2, excretándose a través de las heces por varias semanas. Se ha reportado evidencia de la transmisión fecal-oral aunque se desconoce su alcance (Nouri-Vaskeh \& Alizadeh, 2020). Los médicos deben monitorear los signos y síntomas en el aparato digestivo, específicamente a nivel intestinal y hepático de pacientes con COVID-19 de forma temprana y reforzar la prevención a través de la higiene de manos y de la preparación de los alimentos (Kopel et al., 2020).

Los síntomas gastrointestinales más frecuentes en pacientes con COVID-19 son anorexia y diarrea, seguido de náusea y vómitos, con mayor incidencia de diarrea en adultos que en niños (Y. Zhao et al., 2020). Aunque se ha reportado mayor prevalencia de síntomas gastrointestinales en enfermedad severa no se ha demostrado una correlación entre ambos (Liu et al., 2020). En pacientes con COVID-19, puede haber coexistencia de presentación quirúrgica como apendicitis o bien una imitación de un cuadro quirúrgico por la inflamación mesentérica (Ashcroft et al., 2020).

\section{Manifestaciones en piel y anejos}

Las manifestaciones en piel son infrecuentes, sin embargo, con el conocimiento de las diversas presentaciones en piel, los médicos de primera línea deben incorporar estos signos a su evaluación clínica rutinaria.

El exantema maculopapular acompañado o no de prurito ha sido reportado en uno de cada dos enfermos en España, con un promedio de duración de nueve días y asociado a enfermedad más severa (Galván et al., 2020). Las presentaciones de exantema petequial, purpúrico, urticarial, variceliforme han sido reportados en 
COVID-19 leve a moderado. El exantema tipo eritema multiforme es más común en niños con enfermedad leve (Wollina et al., 2020).

Las erupciones acrales o "dedos de COVID-19" son lesiones similares a los sabañones o también llamado eritema pernio (lesiones inflamatorias producidas por el frío y la humedad). Estas se presentan mayormente en niños y pacientes adultos jóvenes que son asintomáticos o desarrollan una enfermedad leve (Galván et al., 2020). En contraste, las lesiones acro-isquémicas en dedos de manos y pies, con bullas y gangrena seca se presentan en pacientes críticamente enfermos, asociado a un estado de hipercoagulabilidad y coagulación intravascular diseminada (Zhang et al., 2020).

En España se ha observado de que los casos severos de COVID-19 en hombres tenían una alta prevalencia de alopecia androgénica, sugiriendo una vulnerabilidad en el sexo masculino asociada a la sensibilidad a los andrógenos y su actividad inmunosupresora (Goren et al., 2020). Se ha sugerido llamar a esto "signo de Gabrin" en honor al primer médico estadounidense fallecido por COVID-19 quien la padecía siendo sobreviviente de cáncer testicular (Wambier et al., 2020). Dado que la alopecia androgénica tiene una predisposición genética, es importante su identificación clínica en varones como un posible factor de riesgo para sufrir enfermedad severa.

Las lesiones de uñas son raras, aunque se han reportado lesiones descritas como bandas rojas transversales, denominadas "signo media luna roja", considerado un fenómeno transitorio secundario a inflamación vascular (Mendez-Flores et al., 2020).

\section{Manifestaciones genitourinarias}

Se ha reportado un caso de úlceras orales y genitales (úlceras de Lipschütz) en una mujer de 41 años con una presentación leve de COVID-19 en quien se realizó una investigación exhaustiva descartando otras causas, sugiriendo que la infección por SARS-CoV-2 podría desencadenarlo, como sucede para otras infecciones virales (Falkenhain-López et al., 2020).

El tejido genital masculino presenta una alta expresión del receptor ACE2 y existe evidencia reciente, aunque insuficiente de infertilidad masculina en pacientes recuperados de COVID-19. El mecanismo fisiopatológico propuesto es el fallo de la espermatogénesis por la afección directa del virus o por una orquitis causada por elevaciones de temperatura durante los episodios de fiebre y respuestas autoinmunes secundarias (Youssef \& Abdelhak, 2020).
La lesión renal aguda con manifestaciones de proteinuria (44\%), hematuria (26.7\%), elevación de creatinina sérica (15.5\%) y nitrógeno de urea (14.1\%) es relativamente común en la enfermedad severa por COVID-19, prolongando la estancia hospitalaria e incrementando la mortalidad (Cheng et al., 2020). Se considera que la lesión renal es multifactorial, producto de la lesión directa por infección de las células del riñón que expresa el receptor ACE2, lesión hipóxica por sepsis, alteraciones hemodinámicas, coagulación intravascular diseminada y tormenta de citocinas (Asgharpour et al., 2020; Braun et al., 2020).

\section{Manifestaciones neurológicas}

SARS-CoV-2 tiene tropismo por el sistema nervioso central, principalmente a través de la mucosa nasal vía los nervios periféricos utilizando las vías transinápticas, infectando tanto neuronas como neuroglias que expresan el receptor ACE2. La inflamación neuronal en conjunto con la hipoxia prolongada puede producir trastornos neuropsiquiátricos y alteraciones cognitivas agudas y crónicas (Steardo et al., 2020).

La anosmia (pérdida del olfato) y la ageusia (pérdida del gusto) se presentan con mayor frecuencia como síntomas independientes o en asociación con las manifestaciones más comunes de la enfermedad, como fiebre, tos y disnea (Tanasa et al., 2020).

La sintomatología neurológica ha sido asociada a COVID-19, sin embargo, no es específica para esta enfermedad. El SARS-CoV-2 tiene el potencial de acceso directo al sistema nervioso, pero solo se ha detectado en el líquido cefalorraquídeo en dos casos (Chen et al., 2020). En un estudio prospectivo en 239 pacientes en Turquía, se encontraron hallazgos neurológicos en el $34.7 \%$, siendo cefalea el más frecuente (26.7\%), seguido de trastornos del dormir (12.6\%), estado confusional (9.6\%), anosmia (7.5\%), mareos (6.7\%), ageusia (6.7\%), neuralgia glosofaríngea (3.7\%) y neuralgia del trigémino (3.3\%) (Karadas et al., 2020).

Las psicosis agudas han sido relacionadas previamente a infecciones por otros coronavirus. Las presentaciones clínicas más frecuentemente reportadas son delirios estructurados mezclados con características confusionales. Una relación causal entre la infección por SARS-CoV-2 y la aparición de síntomas psicóticos no ha sido establecida, se postula que puede ser resultado de la invasión neuronal, inflamación sistémica, respuesta psicosocial resultante de efectos estresantes de aislamiento prolongado y cambio de la vida durante la pandemia (Huarcaya-Victoria et al., 2020; Parra et al., 2020). 


\section{Manifestaciones inflamatorias y autoinmunes}

En casos severos de COVID-19, las complicaciones inmunológicas como el síndrome de activación de macrófagos, también conocido como linfohistiocitosis hemofagocítica secundaria, es resultado de una liberación incontrolada o "tormenta" de citocinas que causa SDRA y disfunción de órganos en los pacientes que la sufren (Soy et al., 2020). Esta respuesta inmune tiene una patogénesis compleja, con progresión rápida y mortalidad alta, requiriendo un esfuerzo por controlar la respuesta inflamatoria con inmunomoduladores como los corticosteroides y antagonistas de las citocinas IL-1, IL-6 y medidas efectivas para aliviar la inflamación celular en el tejido pulmonar $(\mathrm{Q}$. Ye et al., 2020).

En niños se ha descrito un cuadro hiperinflamatorio con características similares a la enfermedad de Kawasaki y al síndrome de choque tóxico, denominado síndrome inflamatorio multisistémico pediátrico asociado temporalmente con el SARS-CoV-2 (PIMSTS), que ocurre luego de dos a cuatro semanas de la infección aguda. La presentación clínica incluye fiebre, exantema (52\%), inyección conjuntival (45\%) y síntomas no específicos como vómitos, dolor abdominal y diarrea. Los análisis paraclínicos revelan alteraciones inflamatorias con proteína $\mathrm{C}$ reactiva y ferritina elevadas, con evidencia de lesión miocárdica, choque y desarrollo de aneurismas arteriales. En comparación con la enfermedad de Kawasaki, se presenta en niños mayores (media de nueve años versus tres años) y con una mayor elevación de biomarcadores inflamatorios (Whittaker et al., 2020) como se muestra en la Figura 2.

\section{Presentación clínica en adultos mayores}

En adultos mayores la COVID-19 es particularmente severa, con una letalidad de $8 \%$ en el grupo de 70 a 80 años y del $14.8 \%$ en el grupo mayor de 80 años. Dado que la edad avanzada se acompaña de comorbilidades, los adultos mayores tienen más complicaciones y daño de órganos, incluyendo SDRA, lesión renal aguda, lesión cardíaca y disfunción hepática (Nanda et al., 2020). Una revisión sistemática de la literatura encontró que los síntomas predominantes en pacientes con edad media entre 71 a 74 años fueron fiebre, tos seca, disnea, astenia, anorexia, opresión de pecho, diarrea y en menor grado mialgia, faringitis, náusea, mareo, cefalea, dolor abdominal y vómitos (Sacco et al., 2020). En una encuesta de síntomas en mayores de 70 años en Francia, se encontró diferencia significativa en los mayores de 80 años quienes presentaron mayor frecuencia de síntomas como caídas, astenia, delirio y alteración de la conciencia (Annweiler et al., 2020).

\section{Presentación clínica en niños}

Las manifestaciones clínicas en niños difieren de las que presentan los adultos. En una revisión sistemática de la literatura, de un total de 38 estudios en 1,117 pacientes, se estableció el curso de la enfermedad con infección asintomática en $14.2 \%$, leve en $36.3 \%$, moderado $46 \%, 2.1 \%$ severo y crítico $1.2 \%$, con una muerte reportada. El síntoma más prevalente fue la fiebre (47.5\%), seguido de tos seca (41.55\%), síntomas nasales $(11.2 \%)$, diarrea (8.1\%) y nausea/ vómitos (7.1\%). El $36.9 \%$ de los niños presentó neumonía y el $10.9 \%$ una infección limitada al tracto respiratorio superior (de Souza et al., 2020). La recuperación de COVID-19 en niños usualmente ocurre en una a dos semanas. Los casos severos muestran disnea, dificultad respiratoria que progresa a SDRA, choque séptico, acidosis metabólica refractaria, disfunción de la coagulación y fallo orgánico múltiple (Assaker et al., 2020; Shen et al., 2020). Aunque la severidad y mortalidad es menor en los niños, los neonatos y lactantes tienen mayor riesgo de presentar COVID-19 severa, aunque la mortalidad es baja (Raba et al., 2020) ver Tabla 1.

\section{Presentación clínica en el embarazo}

En mujeres embarazadas se ha reportado que el $78 \%$ presenta enfermedad leve o asintomática, mientras que el resto se presentan con enfermedad moderada a severa. El primer grupo presenta síntomas limitados a tos, fiebre, mialgia, faringodinia, opresión de pecho, cefalea, diarrea, nausea, anosmia y ageusia. La presentación moderada a severa incluye tos, disnea, taquipnea (> 30 respiraciones $/ \mathrm{min}$ ), hipoxemia $\left(\mathrm{SatO}_{2}<93 \%\right.$ al aire ambiente) e infiltrados pulmonares en $>50 \%$ del volumen pulmonar en la radiografía de tórax dentro de las 48 horas de presentación (Andrikopoulou et al., 2020). Dado que los síntomas respiratorios en mujeres embarazadas con COVID-19 son leves, es necesario realizar un tamizaje efectivo en la admisión a labor (N. Li et al., 2020). 


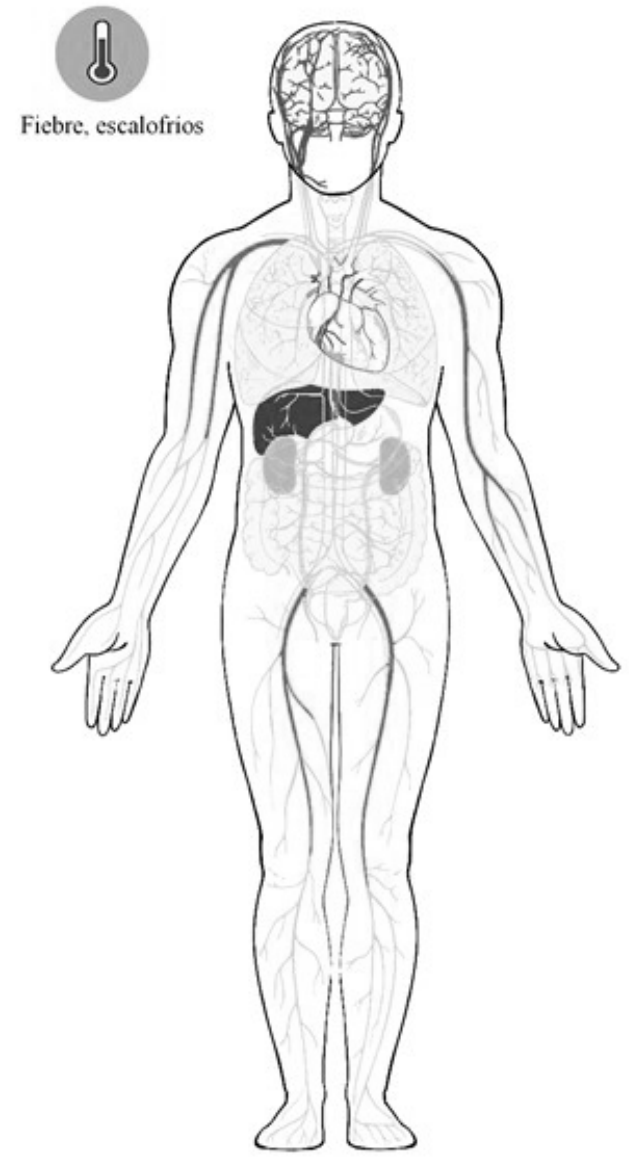

Cefalea, mareos, confusión, neuralgia, ataxia, convulsiones

Ojo rojo, lagrimeo y quemosis

Congestión nasal, rinorrea. anosmia. disgeusia, faringodinia

Tos seca. fatiga, disnea, dolor pleuritico, estertores crepitantes finos $y$ luego estertores y roncus

Dolor precordial, disnea, arritmias. signos de falla cardiaca, hipertensión, $\uparrow$ troponina

Diarrea, náusea, vómitos, dolor abdominal, $\uparrow$ transaminasas

Proteinuria, hematuria, oligoanuria, $\uparrow$ creatinina, $\uparrow$ nitrógeno de urea

Mialgias, artralgias

Rash, eritema pernio ("dedos de COVID-19"), lesiones acroisquémicas.

Hipercoagulabilidad: $\uparrow \mathrm{TP}, \uparrow \mathrm{INR}, \uparrow$ dimero D. $\downarrow$ plaquetas, $\downarrow$ aPTT Hiperinflamación: fiebre, $\uparrow$ ferritina. $\uparrow$ IL-1, $\uparrow$ IL-6

Figura 2. Manifestaciones clínicas y paraclínicas de la COVID-19

\section{COVID-19 y Cáncer}

En una serie de casos en Reino Unido, Italia y España, se reportó que en 204 pacientes con cáncer la fiebre se presentó con mayor frecuencia $(66.7 \%)$, seguido de tos (58.3\%), disnea (39.7\%), fatiga (25\%), mialgia $(11.8 \%)$ y diarrea $(9.8 \%)$. La tasa de complicaciones fue alta, insuficiencia renal aguda y SDRA $(62.7 \%$ y $24 \%$ respectivamente), con una mortalidad estimada del $29 \%$. Se identificó que edad mayor de 65 años y tener más de dos comorbilidades son predictores de mortalidad independientemente del estadiaje tumoral, la malignidad activa o la terapia anti cáncer (Pinato et al., 2020). En una serie de casos en Perú en 69 niños con cáncer, los síntomas más frecuentes fueron fiebre $\mathrm{y}$ tos $(66.7 \%)$ con una presentación moderada-severa en $18.8 \%$ y una mortalidad del $10 \%$ (Montoya et al., 2020).

\section{COVID-19 y trasplante de órgano sólido}

Las manifestaciones clínicas y los hallazgos radiográficos de los pacientes portadores de trasplante de órgano sólido (p.ej. riñón, hígado, pulmón) son similares a los de la población general, aunque presentan con mayor frecuencia síntomas gastrointestinales. En estos individuos la COVID-19 parece ser más grave y tiene una mayor mortalidad (18.4\%) en comparación con la población general (Moosavi et al., 2020).

\section{Coinfecciones e infecciones secundarias}

En un meta-análisis que incluyo 3,834 pacientes, se encontró evidencia de coinfección viral en un $3 \%$, con virus sincitial respiratorio e influenza A como los más comunes y coinfección bacteriana en $7 \%$ de 
Tabla 1

Signos y síntomas comunes y poco comunes de la COVID-19

\begin{tabular}{lll}
\hline Paciente & Comunes & Poco comunes \\
\hline Adultos & $\begin{array}{l}\text { Fiebre, tos seca, fatiga, anosmia, disgeusia, cefalea, } \\
\text { mialgias/artralgias }\end{array}$ & $\begin{array}{l}\text { Conjuntivitis, dolor pleurítico, diarrea, } \\
\text { dolor abdominal, rash, eritema pernio }\end{array}$ \\
Niños & Fiebre, tos seca, rinorrea, diarrea, naúseas, vómitos & Conjuntivitis, rash \\
\hline
\end{tabular}

los casos, principalmente Mycoplasma pneumoniae, Pseudomonas aeuruginosa y Haemophilus influenzae. La mayoría de pacientes (>90\%) recibió antibióticos (Lansbury et al., 2020).

Aunque a la fecha no se ha reportado un incremento de infecciones asociadas a la atención en salud (IAAS) o de la resistencia antimicrobiana en las instituciones que atienden COVID-19, sin embargo se debe fortalecer la vigilancia, las medidas de prevención y control, especialmente la higiene demanos y la optimización y uso racional de antimicrobianos (Ribas et al., 2020).

\section{Coinfección SARS-CoV-2 y Virus de la Inmunodeficiencia Humana (VIH)}

La presentación clínica y pronóstico en personas con coinfección VIH y SARS-CoV-2 son similares a la población general, con predominio de síntomas respiratorios incluyendo tos, esputo y disnea (Byrd et al., 2020; SeyedAlinaghi et al., 2020).

\section{Recuperación y secuelas}

En un estudio de cohorte de 279 pacientes con enfermedad moderada a severa, se encontró que algunos síntomas pueden persistir por muchos meses después del alta, siendo los más frecuentemente reportados fatiga (55\%), disnea (42\%), pérdida de memoria (34\%), trastornos del dormir (31\%) y pérdida de cabello (20\%). Entre los pacientes que trabajaban antes de enfermar, el $69 \%$ habían regresado a laborar al momento de la entrevista, mientras que de los pacientes que practicaban algún deporte de forma regular, el 72\% habían reiniciado la actividad aunque la mitad a una exigencia baja (Garrigues et al., 2020).

\section{Reactivación o reinfección}

Algunos pacientes experimentan lo que parece ser una reactivación de una portación crónica del virus o bien una posible reinfección con efectos potenciales a largo plazo de los fármacos o enfermedades que alteran la respuesta inmune, constituyendo un tema importante en la vigilancia de la enfermedad a nivel de individuo y de la población (Batisse et al., 2020).

\section{Conclusiones}

Como nunca antes había ocurrido para una nueva enfermedad, una riqueza de conocimiento clínico se ha generado a nivel mundial desde enero 2020. Se conoce que la COVID-19 es una enfermedad altamente transmisible en seres humanos y aunque la vía aérea es la principal ruta de transmisión, es posible la transmisión indirecta al encontrarse el virus presente en las heces y también es capaz de permanecer en superficies inertes por horas a días. Hasta la mitad de casos tienen una presentación asintomática, mientras que, en los sintomáticos, la presentación inicial puede ser un cuadro febril acompañado o no de síntomas respiratorios, pudiendo presentar signos y síntomas inespecíficos que se sobreponen y que difieren en niños y adultos. Aunque la enfermedad suele presentarse como una infección respiratoria y evolucionar a neumonía, también puede producir manifestaciones en otros órganos, siendo la hiperinflamación sistémica y SDRA las complicaciones más severas.

Lo principal para los clínicos durante la pandemia (y probablemente por mucho tiempo) es el reconocimiento temprano de la enfermedad y los factores de riesgo asociados a mayor severidad, la confirmación 
del diagnóstico con pruebas confiables, las decisiones sobre el manejo ambulatorio u hospitalario. Para la mejora en la atención de los casos severos, es imprescindible reconocer de forma temprana las complicaciones multiorgánicas, prescribiendo opciones terapéuticas eficaces que reduzcan el riesgo de la mortalidad. Aún falta mucho por conocer sobre la "nueva" enfermedad, pero la creciente evidencia de reportes de reactivación o reinfecciones es motivo de reflexión a enfocarnos en aquellos que se han recuperado, pues como sabiamente dijo Sir William Osler "no existen enfermedades sino enfermos".

\section{Referencias}

Andrikopoulou, M., Madden, N., Wen, T., Aubey, J. J., Aziz, A., Baptiste, C. D., . . Friedman, A. M. (2020). Symptoms and critical illness among obstetric patients with coronavirus disease 2019 (COVID-19) infection. Obstetrics \& Gynecology, 136(2), 291-299. https://doi.org/10.1097/ AOG.0000000000003996

Annweiler, C., Sacco, G., Salles, N., Aquino, J. P., Gautier, J., Berrut, G., . . Gavazzi, G. (2020). National French survey of COVID-19 symptoms in people aged 70 and over. Clinical Infectious Diseases, ciaa792. http://doi.org/10.1093/cid/ ciaa792

Asgharpour, M., Zare, E., Mubarak, M., \& Alirezaei, A. (2020). COVID-19 and kidney disease: Update on epidemiology, clinical manifestations, pathophysiology and management. Journal of the College of Physicians and Surgeons Pakistan, 30(6), 19-25. https://doi.org/10.29271/jcpsp.2020. Supp1.S19

Ashcroft, J., Hudson, V. E., \& Davies, R. J. (2020). COVID-19 gastrointestinal symptoms mimicking surgical presentations. Annals of Medicine and Surgery, 56, 108-109. https://doi.org/10.1016/j. amsu.2020.06.025

Assaker, R., Colas, A. E., Julien-Marsollier, F., Bruneau, B., Marsac, L., Greff, B., ... Dahmani, S. (2020). Presenting symptoms of COVID-19 in children: a meta-analysis of published studies. British Journal of Anaesthesia 125(3), Article e330-e332. https://doi.org/10.1016/j. bja.2020.05.026
Batisse, D., Benech, N., Botelho-Nevers, E., Bouiller, K., Collarino, R., Conrad, A., . . . Wyplosz, B. (2020). Clinical recurrences of COVID-19 symptoms after recovery: viral relapse, reinfection or inflammatory rebound? Journal of Infection, 81(5), 816-846 https://doi.org/10.1016/j. jinf.2020.06.073

Bikdeli, B., Madhavan, M. V., Jimenez, D., Chuich, T., Dreyfus, I., Driggin, E., . . Right Ventricular, F. (2020). COVID-19 and thrombotic or thromboembolic disease: implications for prevention, antithrombotic therapy, and followup: JACC state-of-the-art review. Journal of the American College of Cardiology, 75(23), 29502973. https://doi.org/10.1016/j.jacc.2020.04.031

Braun, F., Lutgehetmann, M., Pfefferle, S., Wong, M. N., Carsten, A., Lindenmeyer, M. T., . . . Huber, T. B. (2020). SARS-CoV-2 renal tropism associates with acute kidney injury. Lancet, 396(10251), 597-598. https://doi.org/10.1016/ S0140-6736(20)31759-1

Byrd, K. M., Beckwith, C. G., Garland, J. M., Johnson, J. E., Aung, S., Cu-Uvin, S., . . Kantor, R. (2020). SARS-CoV-2 and HIV coinfection: clinical experience from Rhode Island, United States. Journal of the International AIDS Society, 23(7), Article e25573. https://doi.org/10.1002/jia2.25573

Center of Disease Control and Prevention. (2020). Infection control guidance for healthcare orofessionals about coronavirus (COVID-19). Recuperado de https://www.cdc.gov/ coronavirus/2019-ncov/hcp/infection-control.html

Chen, X., Laurent, S., Onur, O. A., Kleineberg, N. N., Fink, G. R., Schweitzer, F., \& Warnke, C. (2020). A systematic review of neurological symptoms and complications of COVID-19. Journal of Neurology. https://doi.org/10.1007/s00415-02010067-3

Cheng, Y., Luo, R., Wang, K., Zhang, M., Wang, Z., Dong, L., . . X Xu, G. (2020). Kidney disease is associated with in-hospital death of patients with COVID-19. Kidney International, 97(5), 829-838. https://doi.org/10.1016/j.kint.2020.03.005

Chow, C. B. (2004). Post-SARS infection control in the hospital and clinic. Paediatric Respiratory Reviews, 5(4), 289-295. https://doi.org/10.1016/j. prrv.2004.07.006 
Connors, J. M., \& Levy, J. H. (2020). Thromboinflammation and the hypercoagulability of COVID-19. Journal of Thrombosis and Haemostasis, 18(7), 1559-1561. https://doi. org/10.1111/jth.14849

Cruz, J., Hernández, P., Dueñas, N., \& Salvato, A. (2012). Importancia del método clínico. Revista Cubana de Salud Pública, 38(3), 422-437.

Cucinotta, D., \& Vanelli, M. (2020). WHO declares COVID-19 a pandemic. Acta Biomedica, 91(1), 157-160. https://doi.org/10.23750/abm.v91i1.9397

de Souza, T. H., Nadal, J. A., Nogueira, R. J. N., Pereira, R. M., \& Brandao, M. B. (2020). Clinical manifestations of children with COVID-19: A systematic review. Pediatric Pulmonology, 55(8), 1892-1899. https://doi.org/10.1002/ppul.24885

Dhakal, B. P., Sweitzer, N. K., Indik, J. H., Acharya, D., \& William, P. (2020). SARS-CoV-2 infection and cardiovascular disease: COVID-19 heart. Heart Lung and Circulation, 29(7), 973-987. https://doi. org/10.1016/j.hlc.2020.05.101

Falkenhain-López, D., Agud-Dios, M., Ortiz-Romero, P. L., \& Sánchez-Velázquez, A. (2020). COVID19-related acute genital ulcers. Journal of the European Academy of Dermatology and Venereology. https://doi.org/10.1111/jdv.16740

Galván, C., Catala, A., Carretero, G., RodríguezJimenez, P., Fernández-Nieto, D., RodríguezVilla, A., . . Garcia-Doval, I. (2020). Classification of the cutaneous manifestations of COVID-19: A rapid prospective nationwide consensus study in Spain with 375 cases. British Journal of Dermatology, 183(1), 71-77. https://doi. org/10.1111/bjd.19163

Gao, Z., Xu, Y., Sun, C., Wang, X., Guo, Y., Qiu, S., \& Ma, K. (2020). A systematic review of asymptomatic infections with COVID-19. Journal of Microbiology, Immunology, and Infection. https://doi.org/10.1016/j.jmii.2020.05.001

Garrigues, E., Janvier, P., Kherabi, Y., Bot, A. L., Hamon, A., Gouze, H., . . . Nguyen, Y. (2020). Post-discharge persistent symptoms and healthrelated quality of life after hospitalization for COVID-19. Journal of Infection. https://doi. org/10.1016/j.jinf.2020.08.029
Goren, A., Vano-Galvan, S., Wambier, C. G., McCoy, J., Gomez-Zubiaur, A., Moreno-Arrones, O. M., . . . Washenik, K. (2020). A preliminary observation: Male pattern hair loss among hospitalized COVID-19 patients in Spain - A potential clue to the role of androgens in COVID-19 severity. Journal of Cosmetic Dermatology, 19(7), 15451547. https://doi.org/10.1111/jocd.13443

Goyal, P., Choi, J. J., Pinheiro, L. C., Schenck, E. J., Chen, R., Jabri, A., . . Satlin, M. J. (2020). Clinical characteristics of Covid-19 in New York City. New England Journal of Medicine, 382(24), 2372-2374. https://doi.org/10.1056/ NEJMc2010419

Gravell, R. J., Theodoreson, M. D., Buonsenso, D., \& Curtis, J. (2020). Radiological manifestations of COVID-19: key points for the physician. British Journal of Hospital Medicine, 81(6), 1-11. https:// doi.org/10.12968/hmed.2020.0231

Guan, W., Ni, Z., Hu, Y., Liang, W., Ou, C., He, J., . . Z Zhong, N. (2020). Clinical characteristics of Coronavirus Disease 2019 in China. New England Journal of Medicine, 382(18), 17081720. https://doi.org/10.1056/NEJMoa2002032

He, X., Lau, E. H. Y., Wu, P., Deng, X., Wang, J., Hao, X., . . . Leung, G. M. (2020). Temporal dynamics in viral shedding and transmissibility of COVID-19. Nature Medicine, 26(5), 672-675. https://doi.org/10.1038/s41591-020-0869-5

Hu, L., \& Wang, C. (2020). Radiological role in the detection, diagnosis and monitoring for the coronavirus disease 2019 (COVID-19). European Review for Medical and Pharmacological Sciences, 24(8), 4523-4528. https://doi. org/10.26355/eurrev_202004_21035

Huarcaya-Victoria, J., Meneses-Saco, A., \& LunaCuadros, M. A. (2020). Psychotic symptoms in COVID-19 infection: A case series from Lima, Peru. Psychiatry Research, 293, 113378. https:// doi.org/10.1016/j.psychres.2020.113378

Jacobi, A., Chung, M., Bernheim, A., \& Eber, C. (2020). Portable chest X-ray in coronavirus disease-19 (COVID-19): A pictorial review. Clinical Imaging, 64, 35-42. https://doi.org/10.1016/j. clinimag.2020.04.001 
Karadas, O., Ozturk, B., \& Sonkaya, A. R. (2020). A prospective clinical study of detailed neurological manifestations in patients with COVID-19. Neurological Sciences, 41(8), 1991-1995. https:// doi.org/10.1007/s10072-020-04547-7

Klompas, M., Baker, M. A., \& Rhee, C. (2020). Airborne transmission of SARS-CoV-2: Theoretical considerations and available evidence. Journal of the American Medical Association, 324(5), 441-442. https://doi.org/10.1001/jama.2020.12458

Kopel, J., Perisetti, A., Gajendran, M., Boregowda, U., \& Goyal, H. (2020). Clinical insights into the gastrointestinal manifestations of COVID-19. Digestive Diseases and Sciences, 65(7), 19321939. https://doi.org/10.1007/s10620-02006362-8

Lansbury, L., Lim, B., Baskaran, V., \& Lim, W. S. (2020). Co-infections in people with COVID-19: A systematic review and meta-analysis. Journal of Infection, 81(2), 266-275. https://doi. org/10.1016/j.jinf.2020.05.046

Lauer, S. A., Grantz, K. H., Bi, Q., Jones, F. K., Zheng, Q., Meredith, H. R., . . . Lessler, J. (2020). The incubation period of coronavirus disease 2019 (COVID-19) from publicly reported confirmed cases: Estimation and application. Annals of Internal Medicine, 172(9), 577-582. https://doi. org/10.7326/M20-0504

Li, N., Han, L., Peng, M., Lv, Y., Ouyang, Y., Liu, K., . . . Yang, L. (2020). Maternal and neonatal outcomes of pregnant women with COVID-19 pneumonia: A case-control study. Clinical Infectious Diseases 71(16), 2035-2041. https://doi.org/10.1093/cid/ ciaa352

Li, Q., Guan, X., Wu, P., Wang, X., Zhou, L., Tong, Y., ... Feng, Z. (2020). Early transmission dynamics in Wuhan, China, of novel coronavirus-infected pneumonia. New England Journal of Medicine, 382(13), 1199-1207. https://doi.org/10.1056/ NEJMoa2001316

Liu, J., Cui, M., Yang, T., \& Yao, P. (2020). Correlation between gastrointestinal symptoms and disease severity in patients with COVID-19: A systematic review and meta-analysis. British Medical Journal Open Gastroenterology, 7(1), Article e000437. https://doi.org/10.1136/ bmjgast-2020-000437
Lovato, A., \& de Filippis, C. (2020). Clinical presentation of COVID-19: A systematic review focusing on upper airway symptoms. Ear, Nose \& Throat Journal, 99(9), 569-576,. https://doi. org/10.1177/0145561320920762

Lu, W., Zhang, S., Chen, B., Chen, J., Xian, J., Lin, Y., . . Su, Z. Z. (2020). A clinical study of noninvasive assessment of lung lesions in patients with Coronavirus Disease-19 (COVID-19) by bedside ultrasound. Ultraschall Med, 41(3), 300307. https://doi.org/10.1055/a-1154-8795

Ma, N., Li, P., Wang, X., Yu, Y., Tan, X., Chen, P., . . . Jiang, F. (2020). Ocular manifestations and clinical characteristics of children with laboratoryconfirmed COVID-19 in Wuhan, China. Journal of the American Medical Association Ophthalmology, 138(10), 1079-1086. https://doi. org/10.1001/jamaophthalmol.2020.3690

Mendez-Flores, S., Zaladonis, A., \& Valdes-Rodriguez, R. (2020). COVID-19 and nail manifestation: Be on the lookout for the red half-moon nail sign. International Journal of Dermatology, 59(11), 1414. https://doi.org/10.1111/ijd.15167

Montoya, J., Ugaz, C., Alarcon, S., Maradiegue, E., Garcia, J., Diaz, R., . . Vasquez, L. (2020). COVID-19 in pediatric cancer patients in a resource-limited setting: National data from Peru. Pediatric Blood Cancer, Article e28610. https://doi.org/10.1002/pbc.28610

Moosavi, S. A., Mashhadiagha, A., Motazedian, N., Hashemazar, A., Hoveidaei, A. H., \& Bolignano, D. (2020). COVID-19 clinical manifestations and treatment strategies among solid-organ recipients: a systematic review of cases. Transplant Infectious Diseases, Article e13427. https://doi.org/10.1111/tid.13427

Ministerio de Salud Pública y Asistencia Social. (2020a). Casos COVID-19. Recuperado el 1 de octubre 2020 de https://tablerocovid.mspas.gob.gt

Ministerio de Salud Pública y Asistencia Social. (2020b). Vigilancia epidemiológica COVID-19, Guatemala. Disponible en archivo del Departamento de Epidemiología.

Nanda, A., Vura, N., \& Gravenstein, S. (2020). COVID-19 in older adults. Aging Clinical and Experimental Research, 32(7), 1199-1202. https:// doi.org/10.1007/s40520-020-01581-5 
Nouri-Vaskeh, M., \& Alizadeh, L. (2020). Fecal transmission in COVID-19: A potential shedding route. Journal of Medical Virology, 92(10). https://doi.org/10.1002/jmv.25816

Organización Mundial de la Salud. (2020). Orientaciones técnicas sobre el nuevo coronavirus (2019-nCoV). https:// www.who.int/ es/emergencies/diseases/novelcoronavirus-2019/ technical-guidance

Organización Mundial de la Salud. (2019). Indicadores Básicos 2019: Tendencias de salud en las Américas.

Parra, A., Juanes, A., Losada, C. P., Alvarez-Sesmero, S., Santana, V. D., Marti, I., . . Rentero, D. (2020). Psychotic symptoms in COVID-19 patients. A retrospective descriptive study. Psychiatry Research, 291, 113254. https://doi.org/10.1016/j. psychres.2020.113254

Pinato, D. J., Lee, A. J. X., Biello, F., Segui, E., AguilarCompany, J., Carbo, A., . . Gennari, A. (2020). Presenting features and early mortality from SARS-CoV-2 infection in cancer patients during the initial stage of the COVID-19 pandemic in Europe. Cancers, 12(7). https://doi.org/10.3390/ cancers12071841

Raba, A. A., Abobaker, A., Elgenaidi, I. S., \& Daoud, A. (2020). Novel coronavirus infection (COVID-19) in children younger than one year: A systematic review of symptoms, management and outcomes. Acta Paediatrica, 109(10), 1948-1955. https://doi. org/10.1111/apa.15422

Ribas, R. M., Campos, P. A., Brito, C. S., \& GontijoFilho, P. P. (2020). Coronavirus Disease 2019 (COVID-19) and healthcare-associated infections: Emerging and future challenges for public health in Brazil. Travel Medicine and Infectious Disease, 101675. https://doi. org/10.1016/j.tmaid.2020.101675

Sacco, G., Briere, O., Asfar, M., Guerin, O., Berrut, G., \& Annweiler, C. (2020). Symptoms of COVID-19 among older adults: systematic review of biomedical literature. Geriatrie Psychologie Neuropsychiatrie Vieillissement, 18(2), 135-140. https://doi.org/10.1684/pnv.2020.0863

Salameh, J. P., Leeflang, M. M., Hooft, L., Islam, N., McGrath, T. A., van der Pol, C. B.,... McInnes, M. D. (2020). Thoracic imaging tests for the diagnosis of COVID-19. The Cochrane database of systematic reviews, (9), Article CD013639. https://doi.org/10.1002/14651858.CD013639.pub2 Sattar, Y., Ullah, W., Rauf, H., Virk, H. U. H., Yadav, S., Chowdhury, M., . . Chadi Alraies, M. (2020). COVID-19 cardiovascular epidemiology, cellular pathogenesis, clinical manifestations and management. International Journal of Cardiology Heart \& Vasculature, 29, 100589. https://doi.org/10.1016/j.ijcha.2020.100589

Schiffrin, E. L., Flack, J. M., Ito, S., Muntner, P., \& Webb, R. C. (2020). Hypertension and COVID-19. American Journal of Hypertension, 33(5), 373374. https://doi.org/10.1093/ajh/hpaa057

Seah, I., \& Agrawal, R. (2020). Can the coronavirus disease 2019 (COVID-19) affect the eyes? A review of coronaviruses and ocular implications in humans and animals. Ocular Immunology and Inflammation, 28(3), 391-395. https://doi.org/10.1 080/09273948.2020.1738501

SeyedAlinaghi, S., Ghadimi, M., Hajiabdolbaghi, M., Rasoolinejad, M., Abbasian, L., Nezhad, M. H., . . A Ahmadinejad, Z. (2020). Prevalence of COVID-19-like symptoms among people living with HIV, and using antiretroviral therapy for prevention and treatment. Current HIV Research, 18(5), 373-380. https://doi. org/10.2174/1570162X18666200712175535

Shen, K. L., Yang, Y. H., Jiang, R. M., Wang, T. Y., Zhao, D. C., Jiang, Y., . . Wang, X. F. (2020). Updated diagnosis, treatment and prevention of COVID-19 in children: experts' consensus statement (condensed version of the second edition). World Journal of Pediatrics, 16(3), 232239. https://doi.org/10.1007/s12519-020-00362-4

Siddiqi, H. K., \& Mehra, M. R. (2020). COVID-19 illness in native and immunosuppressed states: A clinical-therapeutic staging proposal. Journal of Heart and Lung Transplantation, 39(5), 405-407. https://doi.org/10.1016/j.healun.2020. 03.012

Soy, M., Keser, G., Atagunduz, P., Tabak, F., Atagunduz, I., \& Kayhan, S. (2020). Cytokine storm in COVID-19: Pathogenesis and overview of anti-inflammatory agents used in treatment. Clinical Rheumatology, 39(7), 2085-2094. https:// doi.org/10.1007/s10067-020-05190-5 
Steardo, L., Steardo, L., Jr., Zorec, R., \& Verkhratsky, A. (2020). Neuroinfection may contribute to pathophysiology and clinical manifestations of COVID-19. Acta Physiologica, 229(3), Article e13473. https://doi.org/10.1111/apha.13473

Sungnak, W., Huang, N., Becavin, C., Berg, M., Queen, R., Litvinukova, M., . . Network, H. C. A. L. B. (2020). SARS-CoV-2 entry factors are highly expressed in nasal epithelial cells together with innate immune genes. Nature Medicine, 26(5), 681-687. https://doi.org/10.1038/s41591-0200868-6

Tanasa, I. A., Manciuc, C., Carauleanu, A., Navolan, D. B., Bohiltea, R. E., \& Nemescu, D. (2020). Anosmia and ageusia associated with coronavirus infection (COVID-19) - what is known? Experimental and Therapeutic Medicine, 20(3), 2344-2347. https://doi.org/10.3892/etm.2020.8808

van Dam, M. V., Ramani, S., \& Ten Cate, O. (2020). Breathing life into bedside teaching in the eraof COVID-19. Medical Teacher, 1-3. https://doi. org/10.1080/0142159x.2020.1798368

Wambier, C. G., Vano-Galvan, S., McCoy, J., GomezZubiaur, A., Herrera, S., Hermosa-Gelbard, A., . . . Goren, A. (2020). Androgenetic alopecia present in the majority of patients hospitalized with COVID-19: The "Gabrin sign". Journal of the American Academy of Dermatology, 83(2), 680682. https://doi.org/10.1016/j.jaad.2020.05.079

Wang, D., Hu, B., Hu, C., Zhu, F., Liu, X., Zhang, J., . .. Peng, Z. (2020). Clinical characteristics of 138 hospitalized patients with 2019 novel coronavirusinfected pneumonia in Wuhan, China. Journal of the American Medical Association, 323(11), 10611069. https://doi.org/10.1001/jama.2020.1585

Whittaker, E., Bamford, A., Kenny, J., Kaforou, M., Jones, C. E., Shah, P., . . PIMS-TS Study Group (2020). Clinical characteristics of 58 children with a pediatric inflammatory multisystem syndrome temporally associated with SARS-CoV-2. Journal of the American Medical Association. https://doi.org/10.1001/jama.2020.10369
Wolff, D., Nee, S., Hickey, N. S., \& Marschollek, M. (2020). Risk factors for Covid-19 severity and fatality: a structured literature review. Infection. https://doi.org/10.1007/s15010-020-01509-1

Wollina, U., Karadag, A. S., Rowland-Payne, C., Chiriac, A., \& Lotti, T. (2020). Cutaneous signs in COVID-19 patients: A review. Dermatologic Therapy, 33(5), Article e13549. https://doi. org/10.1111/dth.13549

Wong, H. Y. F., Lam, H. Y. S., Fong, A. H., Leung, S. T., Chin, T. W., Lo, C. S. Y., ... Ng, M. Y. (2020). Frequency and distribution of chest radiographic findings in patients positive for COVID-19. Radiology, 296(2), E72-E78. doi:10.1148/ radiol.2020201160

Wu, P., Duan, F., Luo, C., Liu, Q., Qu, X., Liang, L., \& Wu, K. (2020). Characteristics of ocular findings of patients with coronavirus disease 2019 (COVID-19) in Hubei Province, China. Journal of the American Medical Association Ophthalmology, 138(5), 575-578. https://doi. org/10.1001/jamaophthalmol.2020.1291

Yang, C., Ma, Q. Y., Zheng, Y. H., \& Yang, Y. X. (2020). Transmission routes of 2019-novel coronavirus (2019-nCoV). Zhonghua Yu Fang Yi Xue Za Zhi, [Chinese Journal of Preventive Medicine] 54(4), 374-377. https://doi.org/10.3760/ cma.j.cn112150-20200216-0016

Ye, Q., Wang, B., \& Mao, J. (2020). The pathogenesis and treatment of the 'Cytokine Storm' in COVID-19. Journal of Infection, 80(6), 607-613. https://doi.org/10.1016/j.jinf.2020.03.037

Ye, Z., Zhang, Y., Wang, Y., Huang, Z., \& Song, B. (2020). Chest CT manifestations of new coronavirus disease 2019 (COVID-19): A pictorial review. European Radiology, 30(8), 4381-4389. https://doi.org/10.1007/s00330-02006801-0

Youssef, K., \& Abdelhak, K. (2020). Male genital damage in COVID-19 patients: Are available data relevant? Asian Journal of Urology. https:// doi.org/10.1016/j.ajur.2020.06.005 
Zhang, Y., Cao, W., Xiao, M., Li, Y. J., Yang, Y., Zhao, J., . . . Li, T. S. (2020). Clinical and coagulation characteristics of 7 patients with critical COVID-2019 pneumonia and acroischemia. Zhonghua Xue Ye Xue Za Zhi, 41(0), Article e006. https://doi.org/10.3760/ cma.j.issn.0253-2727.2020.0006

Zhao, D., Yao, F., Wang, L., Zheng, L., Gao, Y., Ye, J., . . Gao, R. (2020). A Comparative Study on the Clinical Features of Coronavirus 2019 (COVID-19) Pneumonia with other pneumonias. Clinical Infectious Diseases, 71(15), 756-761. https://doi.org/10.1093/cid/ciaa247

Zhao, Y., Cao, Y., Wang, S., Cai, K., \& Xu, K. (2020). COVID-19 and gastrointestinal symptoms. British Journal of Surgery, 107(10). https://doi. org/10.1002/bjs.11821
Zhou, F., Yu, T., Du, R., Fan, G., Liu, Y., Liu, Z., . . . Cao, B. (2020). Clinical course and risk factors for mortality of adult inpatients with COVID-19 in Wuhan, China: A retrospective cohort study. Lancet, 395(10229), 1054-1062. https://doi. org/10.1016/S0140-6736(20)30566-3

Zou, L., Ruan, F., Huang, M., Liang, L., Huang, H., Hong, Z., . . W Wu, J. (2020). SARS-CoV-2 viral load in upper respiratory specimens of infected patients. New England Journal of Medicine, 382(12), 1177-1179. https://doi.org/10.1056/ NEJMc2001737 